\title{
LA ARQUITECTURA DE CONTROL EN EL GOBIERNO CORPORATIVO DE LAS COOPERATIVAS DE AHORRO Y CRÉDITO DEL ECUADOR
}

\author{
THE ARCHITECTURE OF CONTROL IN THE CORPORATE \\ GOVERNANCE OF THE COOPERATIVES OF SAVING \\ AND CREDIT OF ECUADOR
}

\author{
Washington Anibal Altamirano Salazar
}

\begin{abstract}
Resumen
En el Ecuador no existe un modelo de gobierno corporativo, especifico, que incluya mecanismos adecuados de control, para medir y evaluar los riesgos; también para garantizar el uso eficiente de los recursos, con el fin de brindar confianza a un amplio número de stakeholders. En este contexto, el objetivo de la investigación es establecer la incidencia de la arquitectura de control en el gobierno corporativo, en las cooperativas de ahorro y crédito del Ecuador; a su vez, validar un instrumento aplicable para el sector. El instrumento utilizado fue el que elaboró el Banco Interamericano de Desarrollo (BID), el cual fue validado por medio del juicio de expertos mediante el uso de la metodología Delphi. Posteriormente se realizó un estudio empírico, cuantitativo, de tipo correlacional/causal, de corte transversal y no experimental en una muestra de 60 cooperativas. Las técnicas estadísticas empleadas fueron el análisis factorial confirmatorio y el modelo de ecuaciones estructurales para establecer la bondad de ajuste, también la validez y confiabilidad del modelo propuesto. Los resultados determinan que la arquitectura de control tiene una relación significativa y ejerce efectos directos positivos sobre el gobierno corporativo. Se concluye que el instrumento, con base en los lineamientos del BID, es aplicable al sector cooperativo con veintiún variables.
\end{abstract}

\section{Palabras clave}

Arquitectura de control, gobierno corporativo, modelo del Banco Interamericano de Desarrollo, ecuaciones estructurales

\section{Clasificación JEL: G3}

\begin{abstract}
There is no specific corporate governance model in Ecuador that includes adequate control mechanisms to measure and evaluate risks in order to guarantee the efficient use of resources, providing confidence to a wide range of stakeholders. In this context, the aim of this research is to establish the incidence of the control architecture in corporate governance in Ecuador's savings and credit cooperatives, in addition to validating an applicable instrument for the sector. The instrument used was the one developed by the Inter-American Development Bank (IDB), which was validated through expert judgment using the Delphi methodology. Subsequently, an empirical, quantitative, correlational/causal, cross-sectional and non-experimental study was carried out in a sample of 60 cooperatives. The statistical techniques used were the Confirmatory Factor Analysis and the Structural Equation Model to establish the goodness of fit, and the validity and reliability of the proposed model. The results proven that the control architecture has a significant relationship and has direct positive effects on corporate governance; furthermore, it is concluded that the instrument based on the IDB guidelines is applicable to the cooperative sector with 21 variables.
\end{abstract}

\section{Keywords}

Control Architecture, Corporate Governance, Model Inter-American Development Bank, Structural Equations.

JEL Classification: $G 3$ 


\section{Introducción}

En los últimos años, en el sector cooperativo ecuatoriano han ocurrido varios escándalos financieros debido a cierres inesperados de cooperativas. Entre las más recientes están la cooperativa de ahorro y crédito Coopera, en la cual se cometieron delitos de peculado y lavado de activos por un monto de $7.1 \mathrm{mi}-$ llones de dólares. Otra cooperativa es Captec, de Tungurahua, donde se estableció que había créditos que estaban vinculados a directivos por más de 1.5 millones de dólares. Entre las principales causas se encuentra la inadecuada participación democrática de los socios en el gobierno de la cooperativa y en la toma de decisiones, lo cual da pie a que en una cooperativa existan varios actores (stakeholders), quienes hacen que se cree una especial relación de agencia.

Al respecto, Coriat y Weinstein (2011) señalan que la teoría de la agencia es el marco conceptual que analiza las formas de organización económica, su relación y los costos que en ella se incurren. Jensen y Meckling (1976) la definen, por otro lado, como el aspecto contractual entre dos partes, en las que el principal contrata al agente para que administre sus recursos, además de que le asigna poder de decisión. En efecto, en las cooperativas, los socios que son propietarios pueden ser a la vez gestores profesionales y trabajadores; esto hace el que existan distintos intereses que provocan conflictos y que afectan a la gestión de la cooperativa. Los intereses pueden divergir por tres razones fundamentales: cuando existe información imperfecta, por el comportamiento de los agentes y, sobre todo, por la asimetría de la información entre las partes (Charreaux, 1987).

Otro aspecto importante es la falta de un modelo de gobierno corporativo, específico, para el sector cooperativo, que permita establecer, tal como lo manifiestan la Organización para la Cooperación y el Desarrollo
Económicos (OCDE) (2004) y el Banco Interamericano de Desarrollo (BID) (2013), los procedimientos, procesos o el sistema por el que una organización es dirigida y controlada. El gobierno corporativo busca la transparencia en las actividades ordinarias de las empresas, así como el establecimiento de normas que ayuden a su control (Ospina, 2013). Por ello, es el hilo conductor entre las prácticas empresariales sostenibles y la protección de los recursos culturales, ambientales y colectivos de la comunidad" (Buitrago y Betancourt, 2013:).

Desde los inicios del gobierno corporativo, tanto de manera académica (surgió en 1976 de la mano de Michael Jensen y William Meckling, tal como señalan Mendes, Núñez y Oneto -2009-), así como práctica empresarial (empezó en Estados Unidos en 1978 gracias al American Law Institute), se han elaborado códigos, leyes, principios, informes y lineamientos pero todos enfocados en las empresas donde prima el capital. En Europa, por ejemplo, la bolsa de valores crea en 1992 el comité para la realización del informe Cadbury a fin de dar respuesta a la falta de controles (Alonso y Da Silva, 2010). En 1999, la OCDE propone seis principios de gobierno corporativo y brinda, para ello, un marco de referencia (OCDE, 2004). En 2002, los senadores Paul Sarbanes y Michael Oxley presentaron -ante el Congreso de Estados Unidos - la ley Sarbanes Oxley con el propósito de regular a las empresas y los constantes escándalos financieros (García, Cortez y Rodríguez, 2009). Esta ley no exigía la creación de un código de conducta y ética que rija a toda la empresa, más sí recomendaba crearlo, con el fin de fomentar la transparencia en sus operaciones (Gómez y Zapata, 2013). De nuevo en Europa, específicamente en España, se publicó el Código Olivencia en 1998, el Código Aldama en 2003 y el Códi- 
go Unificado del Buen Gobierno Corporativo en 2006. Ya en América Latina, el Banco de Desarrollo de América Latina, con la colaboración de la Corporación Andina de Fomento (CAF), publicó en 2004 los lineamientos para un buen gobierno corporativo, que fueron actualizados 2012.

La inexistencia de un modelo propio para el sector ha impedido que se haga una adecuada implementación y posterior medición del gobierno corporativo en las cooperativas. Esta situación ha acarreado que haya muchos conflictos de intereses y que, por su defensa, se tomen decisiones que generen altos riesgos. Esto ha ocasionado la quiebra de varias cooperativas, inclusive de manera fraudulenta, como se explicó anteriormente.

En este sentido, la arquitectura de control es un elemento esencial en el buen gobierno corporativo por cuanto agrupa los aspectos relacionados con el sistema de control interno, el sistema de administración de riesgos y el control externo o posterior (Banco Interamericano de Desarrollo, 2013). Sin embargo, dichos riesgos no son detectados a tiempo, de manera preventiva, pues los métodos de control de los recursos que utilizan las cooperativas y los organismos de control no son eficientes; tampoco se ajustan a la realidad de este sector.

En relación con el control y los riesgos, el Comité de Organizaciones Patrocinadoras ( COSO, por sus siglas en inglés, que significan Committee of Sponsoring Organizations of the Treadway Commission), emitio en 1992 un informe que tiene como objetivo mejorar la calidad de la información financiera, mediante el establecimiento de controles internos efectivos y de buen gobierno corporativo (Ambrosone, 2007). Este informe fue ampliado en el año 2004 y se denominó COSO II, Enterprise Risk Management (ERM, por sus siglas en inglés), en el que se define un marco conceptual común que se constituye en una visión integradora del control interno por medio de la administración de los riesgos empresariales (Vaca y Casanova, 2014).Este informe proporciona una guía a la alta gerencia, accionistas y directivos en cuanto a la administración de los riesgos desde la definición de estrategia hasta su ejecución. Se destaca la importancia de la relación entre la estrategia y el desempeño de la organización (PricewaterhouseCoopers, 2017).

En todo este contexto la determinación de un modelo de gobierno corporativo, para el sector que incluya mecanismos de control adecuados, permitirá establecer un conjunto de prácticas que guíen el comportamiento entre la administración de la cooperativa y los agentes económicos que tengan algún interés. Esta filosofía corporativa conduce a que se midan y evalúen los riesgos, con el objeto de garantizar el uso eficiente de los recursos y brindar confianza a un amplio número de partes interesadas. Para ello, se crean sistemas idóneos de control interno, sistemas de administración de riesgos y el control externo con base en las auditorías que sirvan para fijar alertas y prevenir estos sucesos.

Por esta razón, los directivos de las cooperativas buscan cómo medir el buen gobierno corporativo a fin de establecer los medios a través de los cuales se puede prevenir y corregir un posible conflicto de intereses entre las partes (Crespi y Gispert, 1999) $y$, de esta manera, asentar mecanismos de control mediante una adecuada arquitectura de control. Esta situación les permitirá mejorar su imagen, proveyendo de un ambiente de seguridad a sus asociados, para que puedan invertir y beneficiarse de sus servicios. Por otro lado, este modelo favorecerá la estandarización de la gestión en este tipo de organizaciones y el establecimiento de mecanismos de control óptimos.

En efecto, la investigación ayudará a que las cooperativas dispongan de un ins- 
trumento validado y confiable para medir si cuentan con un buen gobierno corporativo y una apropiada arquitectura de control. Además de determinarla incidencia entre las variables, para comprobar la siguiente hipótesis.

$\mathrm{H}_{1}$ - La arquitectura de control incide de manera positiva en el gobierno corporativo de las cooperativas de ahorro y crédito.

Para conseguir este objetivo y demostrar la hipótesis, en la primera parte del ar-

\section{Metodología}

La presente investigación se llevó a cabo en dos etapas: en la primera se emplearon técnicas cualitativas por medio de la validación del instrumento desarrollado por el BID, junto con la CAF, y que fue actualizada en 2012. Dicha encuesta contiene cinco dimensiones: derechos y trato de accionistas, asamblea general, tículo se detalla la metodología utilizada en el desarrollo del trabajo. En el segundo apartado se hace una revisión del marco teórico, una breve descripción de las variables y de los estudios realizados con anterioridad en relación con estas. En tercer lugar, se presentan los resultados mediante el análisis de los datos obtenidos a través de técnicas estadísticas. Para finalizar, se muestran las conclusiones y las referencias bibliográficas.

directorio, arquitectura de control y transparencia e información financiera y no financiera. Estas áreas agrupan 43 lineamientos y 133 recomendaciones conforme se expone en la tabla 1. De las cinco dimensiones, cuatro están relacionadas con el gobierno corporativo, y una, con la arquitectura de control (BID, 2013).

Tabla 1. Lineamientos BID del gobierno corporativo

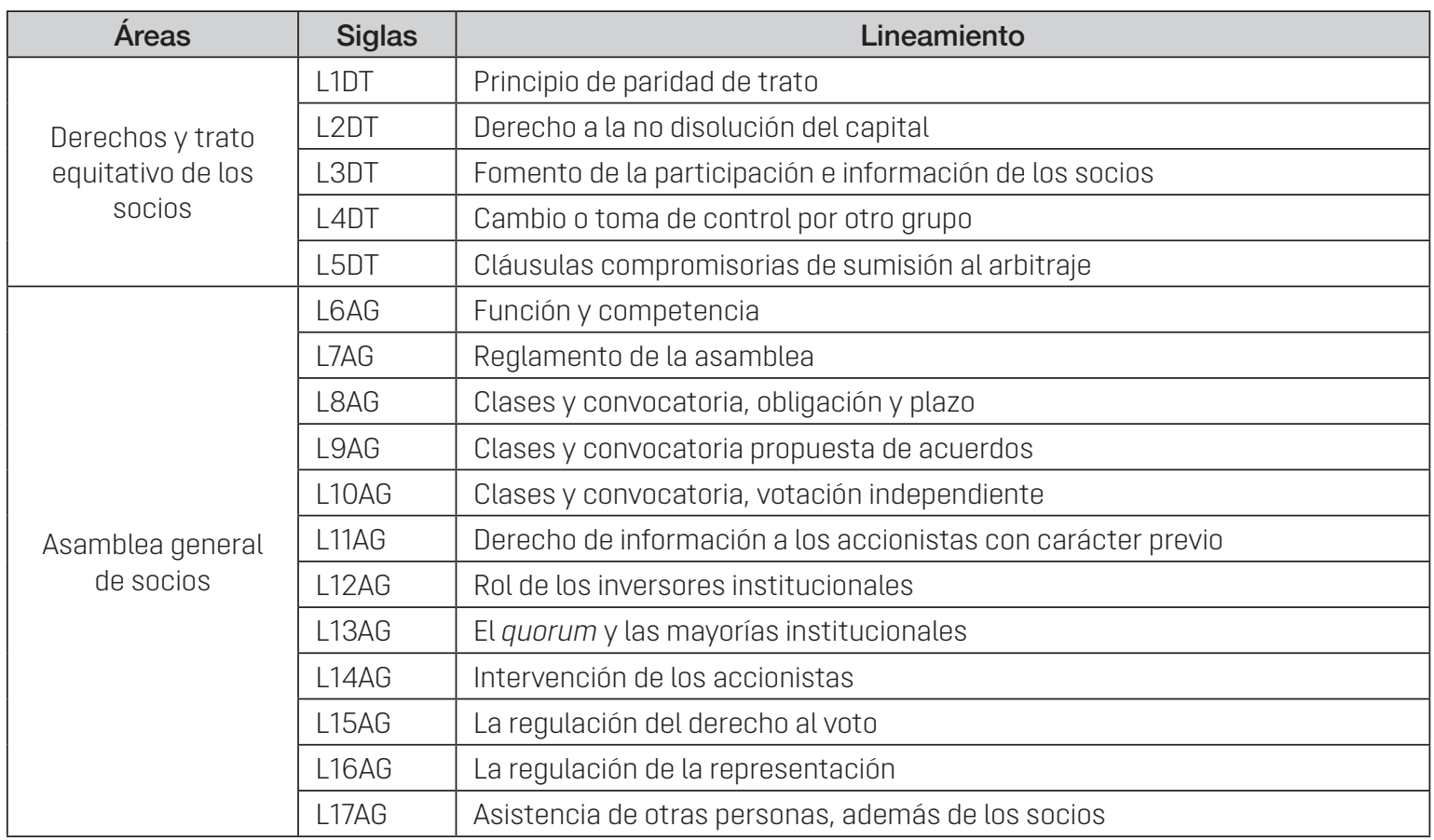




\begin{tabular}{|c|c|c|}
\hline \multirow{15}{*}{$\begin{array}{l}\text { El directorio (conse- } \\
\text { jo de administración) }\end{array}$} & L18DI & Necesidad de tener un directorio \\
\hline & L19DI & $\begin{array}{l}\text { Atribución al directorio de las funciones de definición estratégica, supervi- } \\
\text { sión, control, gobierno y administración }\end{array}$ \\
\hline & L20DI & Reglamento del directorio \\
\hline & L21DI & Dimensión del directorio \\
\hline & L22DI & Categorías y miembros del directorio \\
\hline & L23DI & Nombramiento \\
\hline & L24DI & Directores externos independientes \\
\hline & L25DI & Cese de directores \\
\hline & L26DI & Deberes y derechos \\
\hline & L27DI & Conflictos de interés y operaciones \\
\hline & L28DI & Retribución de los directores \\
\hline & L29DI & Organización del directorio \\
\hline & L30DI & El ejecutivo principal y la alta gerencia \\
\hline & L31DI & Dinámica del directorio \\
\hline & L32DI & Comités del directorio \\
\hline \multirow{6}{*}{$\begin{array}{l}\text { Arquitectura } \\
\text { de control }\end{array}$} & L33AC & El ambiente de control \\
\hline & L34AC & La gestión de riesgos \\
\hline & L35AC & El sistema de control interno \\
\hline & L36AC & $\begin{array}{l}\text { La información y comunicación del sistema de gestión de riesgos y del con- } \\
\text { trol interno }\end{array}$ \\
\hline & L37AC & Labor de auditoría interna \\
\hline & L38AC & Labor de auditoría externa \\
\hline \multirow{5}{*}{$\begin{array}{l}\text { Transparencia } \\
\text { e información } \\
\text { financiera y no } \\
\text { financiera }\end{array}$} & L39TI & Política de revelación de información \\
\hline & L40TI & Información a los mercados \\
\hline & L41TI & Estados financieros \\
\hline & L42TI & Información sobre acuerdos entre socios \\
\hline & L43TI & Informe anual del gobierno corporativo \\
\hline
\end{tabular}

Fuente: elaboración propia.

Para determinar la validez y fiabilidad del instrumento se utilizó el método de validación a través del juicio de expertos, que consistió en solicitar a quince personas, entre ellos profesionales, gerentes y docentes, un juicio u opinión del instrumento, o de un aspecto en concreto (Cabero y Llorente, 2013). A fin de garantizar los resultados, se empleó el método Delphi, el cual consiste, según De Arquer (1995), en que cada experto haga la evaluación de manera individual y luego reciban las medianas obtenidas para que reconsideren su juicio hasta que se llegue a un consenso. Esta técnica permitió conseguir un alto nivel de interacción entre los expertos, evitando, así, las desventajas de la dinámica grupal conforme lo establecen Van der FelsKlerx, Goossens, Saatkamp y Horst (2002).

El trabajo de los expertos estribó en determinar la representatividad, comprensión, interpretación y claridad de cada pregunta. Para el efecto se usó una escala del 1 al 3, con diferente significación. Para analizar la representatividad, "1" indicó "nada representativo", y "3", "muy representativo". Para la comprensión, "1" señala que la pregunta resulta incomprensible, y "3", entendible; en la interpretación, en cambio, el "1" indica que 
puede tener varias interpretaciones, y el " 3 ", que tiene una única interpretación. Por último, y con respecto a la claridad, el "1" quiere decir nada claro, y el "3" conciso (Crespo, D Ambrosio, Racines y Castillo, 2016).

En la segunda etapa se realizó una validación cuantitativa mediante un estudio empírico, de tipo correlacional/causal, de corte transversal y no experimental (Hernández, Fernández y Baptista, 2010). En una muestra de 60 cooperativas para su cálculo se echó mano de la fórmula del método de estimación simple para el muestreo irrestricto aleatorio (Calero, 2003). Los parámetros definidos fueron: población (N) de 146 cooperativas pertenecientes a los segmentos 1 , 2 y 3 de la clasificación de la Superintendencia de Economía Popular y Solidaria debido a que los tres segmentos representan el 87.65 $\%$ en relación con el monto de los activos totales, nivel de significación $(\boldsymbol{\alpha}) 0.05$, error (d) 0.10 , valor de la probabilidad $(P) 0.5$.

Una vez establecida la muestra, se determinó la cantidad de cooperativas por segmento, para lo cual se aplicó la fórmula del muestreo por estratos por asignación proporcional (Calero, 2003). Se obtuvo como resultado 12 cooperativas para el segmento 1; 16 para el segmento 2, y 32 para el segmento 3. A fin de garantizar la selección aleatoria de la muestra, se empleó la tabla de números aleatorios de Kendall y Babington (1939).

Una vez validado el instrumento, fue aplicado en la muestra seleccionada. Los datos fueron sometidos a una evaluación multivariante. Las técnicas estadísticas empleadas fueron el análisis factorial confirmatorio (AFC) y el modelo de ecuaciones estructurales (SEM, por sus siglas en inglés). El objetivo: determinar la bondad de ajuste, aparte de la validez y confiabilidad del modelo de medición propuesto. Además, se calcularon los estadísticos alpfa de Cronbach, fiabilidad compuesta o coeficiente omega y la varianza extraída. Se usó el software es- tadístico SPSS, versión 24, y el programa Amos, versión 22.

Con respecto al sistema de ecuaciones estructurales, se trata de una herramienta avanzada de análisis multivariante, ya que se sirve de un conjunto de ecuaciones de regresión lineal diferentes pero independientes, para analizar las relaciones de las variables latentes consideradas en un modelo (Armario y Cossío, 2001).. Se denominan variables latentes aquellas que no pueden ser observadas directamente, sino a través de variables observadas o indicadores, conforme fueron tratadas las dos variables objeto de estudio de esta investigación. El modelo propuesto es de tipo recursivo porque la causalidad entre las variables tiene una dirección única.

Mediante un modelo gráfico, denominado diagrama de camino (diagrama PATH), se representaron las relaciones entre las variables por medio de cargas estimadas. Para ello se usó la minimización de una función de discrepancia entre la matriz de varianzas y covarianzas. Con el objeto de estimar los parámetros del modelo, se usó el método de máxima verosimilitud (maximun likelihood estimation - MLE, por sus siglas en inglés-) por cuanto se emplearon escalas multiítems y, según Anderson y Gerbing (1982), este método proporciona estimadores que se ajustan adecuadamente tanto en la consistencia interna como en la externa. Posteriormente se debería transformar el gráfico en un conjunto de ecuaciones para especificar las relaciones entre las variables latentes y sus indicadores. Sin embargo, el programa informático utilizado (Amos, versión 22) desarrolla directamente las ecuaciones estructurales a partir del modelo gráfico. Por esta razón, no se recurrirá al lenguaje analítico.

Para realizar la evaluación de los resultados, se revisaron tres aspectos: a) medidas de ajuste global del modelo; b) medidas de ajuste incremental de modelo y c) medidas de ajuste de parsimonia. 


\section{Resultados}

\section{Validación cualitativa}

Con respeto a la validación de este modelo, el instrumento inicial tiene 43 lineamientos, la validación por expertos eliminó

siete y se obtuvo como resultado 36 lineamientos válidos. Ello se demuestra en la tabla 2.

Tabla 2. Resultados de la validación de expertos

\begin{tabular}{|c|c|c|c|}
\hline Áreas & Siglas & Lineamiento & Observaciones \\
\hline \multirow{5}{*}{$\begin{array}{l}\text { Derechos y trato } \\
\text { equitativo de los } \\
\text { socios }\end{array}$} & L1DT & Principio de paridad de trato & \\
\hline & L2DT & Derecho a la no disolución del capital & \\
\hline & L3DT & Fomento de la participación e información de los socios & \\
\hline & L4DT & Cambio o toma de control por otro grupo & \\
\hline & L5DT & Cláusulas compromisorias de sumisión al arbitraje & \\
\hline \multirow{12}{*}{$\begin{array}{l}\text { Asamblea general } \\
\text { de socios }\end{array}$} & L6AG & Función y competencia & \\
\hline & L7AG & Reglamento de la asamblea & \\
\hline & L8AG & Clases y convocatoria, obligación y plazo & \\
\hline & L9AG & Clases y convocatoria propuesta de acuerdos & \\
\hline & L10AG & Clases y convocatoria, votación independiente & \\
\hline & L11AG & $\begin{array}{l}\text { Derecho de información a los accionistas con carácter } \\
\text { previo }\end{array}$ & \\
\hline & L12AG & Rol de los inversores institucionales & Eliminado \\
\hline & L13AG & El quorum y las mayorías institucionales & Eliminado \\
\hline & L14AG & Intervención de los accionistas & Eliminado \\
\hline & L15AG & La regulación del derecho al voto & \\
\hline & L16AG & La regulación de la representación & Eliminado \\
\hline & L17AG & Asistencia de otras personas, además de los socios & \\
\hline \multirow{15}{*}{$\begin{array}{l}\text { El directorio } \\
\text { (consejo de } \\
\text { administración) }\end{array}$} & L18DI & Necesidad de tener un directorio & \\
\hline & L19DI & $\begin{array}{l}\text { Atribución al directorio de las funciones de definición estra- } \\
\text { tégica, supervisión, control, gobierno y administración }\end{array}$ & \\
\hline & L20DI & Reglamento del directorio & \\
\hline & L21DI & Dimensión del directorio & \\
\hline & L22DI & Categorías y miembros del directorio & Eliminado \\
\hline & L23DI & Nombramiento & \\
\hline & L24DI & Directores externos independientes & \\
\hline & L25DI & Cese de directores & \\
\hline & L26DI & Deberes y derechos & Eliminado \\
\hline & L27DI & Conflictos de interés y operaciones & \\
\hline & L28DI & Retribución de los directores & \\
\hline & L29DI & Organización del directorio & \\
\hline & L30DI & El ejecutivo principal y la alta gerencia & \\
\hline & L31DI & Dinámica del directorio & \\
\hline & L32DI & Comités del directorio & \\
\hline
\end{tabular}




\begin{tabular}{|c|c|c|c|}
\hline \multirow{6}{*}{$\begin{array}{l}\text { Arquitectura de } \\
\text { control }\end{array}$} & L33AC & El ambiente de control & \\
\hline & L34AC & La gestión de riesgos & \\
\hline & L35AC & El sistema de control interno & \\
\hline & L36AC & $\begin{array}{l}\text { La información y comunicación del sistema de gestión de } \\
\text { riesgos y del control interno }\end{array}$ & \\
\hline & L37AC & Labor de auditoría interna & \\
\hline & L38AC & Labor de auditoría externa & \\
\hline \multirow{5}{*}{$\begin{array}{l}\text { Transparencia e in- } \\
\text { formación financie- } \\
\text { ra y no financiera }\end{array}$} & L39TI & Política de revelación de información & \\
\hline & L40TI & Información a los mercados & Eliminado \\
\hline & L41TI & Estados financieros & \\
\hline & L42TI & Información sobre acuerdos entre socios & \\
\hline & L43TI & Informe anual del gobierno corporativo & \\
\hline
\end{tabular}

Fuente: Elaboración propia.

\section{Validación cuantitativa}

El instrumento validado fue aplicado en la muestra que se seleccionó y los datos se estudiaron mediante el análisis factorial confirmatorio y el modelo de ecuaciones estructurales. Para el efecto se tomó en cuenta la limitación que Armario y Cossío (2001) indicaron, y para su corrección, la recomendación de Babin y Boles (1998), y de Mackenzie, Podsakoff y Ahearne (1998). Los resultados de la depuración de las escalas se presentan en la tabla 3.

Tabla 3. Escalas de medida de las dimensiones de los lineamientos del BID-CAF

\begin{tabular}{|l|l|l|l|l|l|l|}
\hline Dimensiones & $\begin{array}{c}\text { Ítems } \\
\text { iniciales }\end{array}$ & $\begin{array}{c}\text { Ítems } \\
\text { finales }\end{array}$ & $\begin{array}{c}\text { Alfa de } \\
\text { Cronbach }\end{array}$ & $\begin{array}{c}\text { Fiabilidad de } \\
\text { constructo }\end{array}$ & $\begin{array}{l}\text { Varianza } \\
\text { extraída }\end{array}$ & Observaciones \\
\hline DT & 5 & 3 & 0.666 & 0.685 & 0.429 & Eliminados DT4-5 \\
\hline AG & 8 & 5 & 0.572 & 0.597 & 0.251 & Eliminados AG10-15-17 \\
\hline DI & 13 & 6 & 0.725 & 0.743 & 0.335 & $\begin{array}{l}\text { Eliminados } \\
\text { Dl19-20-21-25-27-29-30 }\end{array}$ \\
\hline AC & 6 & 4 & 0.734 & 0.742 & 0.434 & Eliminados AC33-37 \\
\hline TI & 4 & 3 & 0.788 & 0.806 & 0.590 & Eliminados TI-42 \\
\hline Total & 36 & 21 & & & & \\
\hline
\end{tabular}

Fuente: elaboración propia.

Tal como se observa se han eliminado quince items con relación a la escala inicial por su baja significación estadística y se obtuvo una escala compuesta de 21 ítems. Con respecto a los niveles de consistencia interna, la mayoría de las escalas presentan niveles adecuados, a excepción de la dimensión "asamblea general" (AG). Sin embargo, con base en la teoría, se considera relevante su análisis.
Una de las particularidades de este modelo es la importancia que se le brinda a la "arquitectura de control", por cuanto es una nueva dimensión con respecto a los modelos existentes.

Por esta razón, aparte de la validación del modelo, se consideró importante hacer un análisis de la relación que tiene la "arquitectura de control" con las demás dimensiones del modelo. 


\section{Análisis multivariante entre la arquitectura de control y el gobierno corporativo}

Para el efecto, se determinó que los datos son reflectivos, con la finalidad de garantizar el método estadístico por utilizar. Se obtuvo un alpfa de Cronbach superior al valor aceptable de 0.70, establecido por Luque (1997).

Partiendo de este análisis, se propone el siguiente modelo que mide el gobier- no corporativo, y agrega la incidencia de la dimensión "arquitectura de control" en las cuatro dimensiones de gobierno corporativo que fueron establecidas en los lineamientos del BID. Esto se ilustra en la figura 1.

Figura 1. Modelo propuesto de gobierno corporativo BID-CAF.

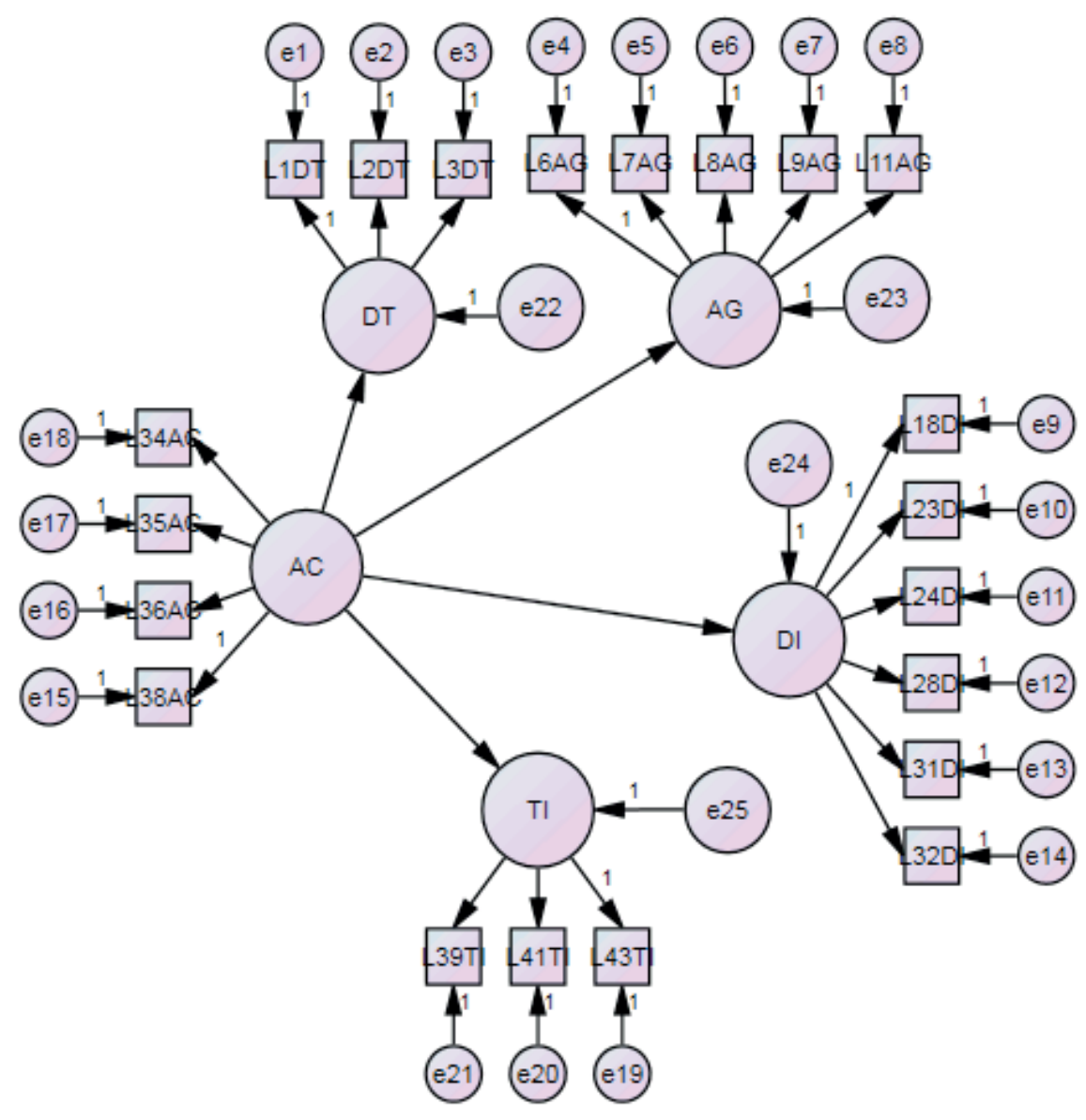

Fuente: elaboración propia

Posteriormente, se llevó a cabo un análisis multivariante a través del modelo de ecuaciones estructurales. En la figura 2 se presentan los resultados. Entre estos se establece que existe una correlación positiva y significativa entre las dimensiones AC-DT,
AC-DI y AC-TI, pues sus indicadores de 0.63 , 0.93 y 0.49 , respectivamente, superan el nivel de 0.40 señalado por Hair et al. (1995). Con respecto a la correlación entre las dimensiones AC-AG, (0.21) no es significativa, sin embargo, sí positiva. 
Figura 2. Resultados del modelo propuesto de gobierno corporativo BID-CAF.

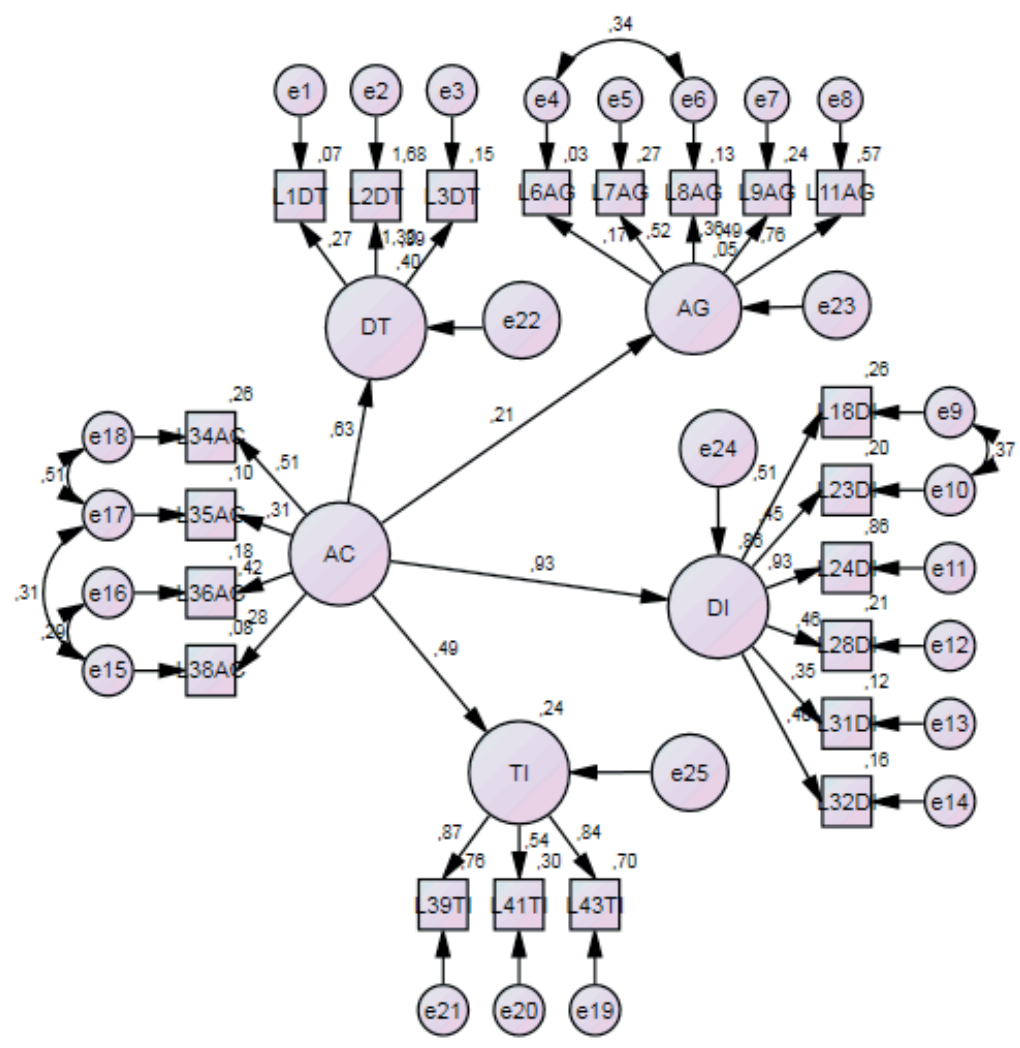

Fuente: elaboración propia.

Con respecto a las estimaciones de la bondad de ajuste del modelo que se expusieron en la tabla 4, los indicadores señalan que algunos de ellos, como el CMINY RMRS, están dentro de los parámetros establecidos, en tanto que los indicadores RMSEA, AGFI Y CFI están muy cerca de los estándares. Con esto se confirman una estimación aceptable para el ajuste del modelo.

Tabla 4. Bondad de ajuste del modelo propuesto

\begin{tabular}{|l|l|l|l|}
\hline \multicolumn{1}{|c|}{ Bondad de ajuste } & \multicolumn{1}{|c|}{$\begin{array}{c}\text { Escala } \\
\text { propuesta }\end{array}$} & Niveles de aceptación & \multicolumn{1}{c|}{ Autor } \\
\hline Medidas de ajuste global & & & \\
\hline Índice de bondad de ajuste (GFI) & 0.627 & Superior a 0.90 & Jöreskog y Sörbom, 1986 \\
\hline Residuo cuadrado medio de la raíz (RMSR) & 0.089 & Valor más próximo a cero & Jöreskog y Sörbom, 1986 \\
\hline $\begin{array}{l}\text { Error de aproximación cuadrático medio } \\
\text { (RMSEA) }\end{array}$ & 0.178 & Valores inferiores a 0.08 & Steiger, 1990 \\
\hline Medidas de ajuste incremental & & & \\
\hline Índice de bondad de ajuste (AGFI) & 0.521 & Superior a 0.90 & Jöreskog y Sörbom, \\
\hline Medidas de ajuste de parsimonia & & & \\
\hline Chi cuadrado normada (CMIN/DF) & 2.859 & Valores entre 2 y hasta 5 & Jöreskog y Sörbom, 1989 \\
\hline
\end{tabular}

Fuente: elaboración propia. 


\section{Modelo propuesto}

Con estos resultados se puede afirmar que la arquitectura de control incide de manera aceptable en el gobierno corporativo de las cooperativas a través de sus dimensiones $D T, A G, D I$ y TI. Así, se puede contrastar que la hipótesis $\mathrm{H}_{1}$ se confirma positivamente. Es decir: se afirma que la arquitectura de con- trol tiene una relación significativa y ejerce efectos directos positivos sobre el gobierno corporativo. Además, también se puede afirmar que el modelo de medida propuesto, sobre la base de los lineamientos del BID-CAF, es aplicable al sector cooperativo con 21 variables, conforme se detalla en la tabla 5.

Tabla 5. Lineamientos BID-CAF validados

\begin{tabular}{|c|c|c|c|}
\hline Áreas & N. ${ }^{\circ}$ & Siglas & Lineamiento \\
\hline \multirow{3}{*}{$\begin{array}{l}\text { Derechos y trato equita- } \\
\text { tivo de los socios }\end{array}$} & 1 & L1DT & Principio de paridad de trato \\
\hline & 2 & L2DT & Derecho a la no disolución del capital \\
\hline & 3 & L3DT & Fomento de la participación e información de los socios \\
\hline \multirow{5}{*}{$\begin{array}{l}\text { Asamblea general de } \\
\text { socios }\end{array}$} & 4 & L6AG & Función y competencia \\
\hline & 5 & L7AG & Reglamento de la asamblea \\
\hline & 6 & L8AG & Clases y convocatoria, obligación y plazo \\
\hline & 7 & L9AG & Clases y convocatoria propuesta de acuerdos \\
\hline & 8 & L11AG & Derecho de información a los accionistas con carácter previo \\
\hline \multirow{6}{*}{$\begin{array}{l}\text { El directorio (consejo de } \\
\text { administración) }\end{array}$} & 9 & L18DI & Necesidad de tener un directorio \\
\hline & 10 & L23DI & Nombramiento \\
\hline & 11 & L24DI & Directores externos independientes \\
\hline & 12 & L28DI & Retribución de los directores \\
\hline & 13 & L31DI & Dinámica del directorio \\
\hline & 14 & L32DI & Comités del directorio \\
\hline \multirow{4}{*}{ Arquitectura de control } & 15 & L34AC & La gestión de riesgos \\
\hline & 16 & L35AC & El sistema de control interno \\
\hline & 17 & L36AC & $\begin{array}{l}\text { La información y comunicación del sistema de gestión de ries- } \\
\text { gos y del control interno }\end{array}$ \\
\hline & 18 & L38AC & Labor de la auditoría \\
\hline \multirow{3}{*}{$\begin{array}{l}\text { Transparencia e infor- } \\
\text { mación financiera y no } \\
\text { financiera }\end{array}$} & 19 & L39TI & Política de revelación de información \\
\hline & 20 & L41TI & Estados financieros \\
\hline & 21 & L43TI & Informe anual de gobierno corporativo \\
\hline
\end{tabular}

Fuente: elaboración propia.

\section{Discusión y conclusiones}

Los resultados de esta investigación permiten determinar que la arquitectura de control incide de manera positiva en el gobierno corporativo de las cooperativas de ahorro y crédito, en sus dimensiones DT, AG, DI y TI. Así, se puede contrastar que la hipótesis $H_{1}$ se con- firma positivamente. Es decir: se afirma que la arquitectura de control tiene una relación significativa y ejerce efectos directos positivos sobre el gobierno corporativo.

Esta investigación demuestra que la arquitectura de control y el gobierno corpo- 
rativo van de la mano; mientras más eficientes son los controles establecidos, mejores son los niveles de gobierno corporativo. Dicha situación deja claro el criterio que proponen Crespi y Gispert (1999) sobre que el buen gobierno corporativo ayuda a prevenir y a corregir un posible conflicto de intereses entre todas las partes relacionadas. Otro aspecto relevante que se debe apuntar es la confirmación de lo que señala el BID (2013), en cuanto a que una adecuada arquitectura de control sirve para evitar que se tomen decisiones que generen fuertes riesgos financieros, que pueden ocasionar, a su vez, hasta la quiebra de este tipo de organizaciones,

\section{Referencias}

Alonso, M. D., Da Silva, J. (2010). Códigos de buen gobierno corporativo en Iberoamérica: análisis comparativo entre Brasil y México. Recuperado de http://www.redalyc. org/articulo.oa?id=337228641006

Ambrosone, M. (mayo de 2007). La administración del riesgo empresarial: una responsabilidad de todosEl enfoque COSO. Recuperado de http://ayhconsultores.com/img/ coso.pdf

Anderson, J., \& Gerbing, D. (1982). Some Methods for Respecifying Measurement Models to Obtain Unidimensional Construct Measurement. Journal of Marketing Research, 19(4), 453-460.

Armario, E.; Cossío, F. (2001). La orientación al mercado y el rendimiento empresarial: el caso de la banca comercial española. Cuadernos de Gestión, 1 (1), 33-66.

Babin, B., \& Boles, J. (1998). Employee Behaviour in a Service Environment: A Model and Test of Potencial Differences between Men and Women. Journal of Marketing, 62(2), 77-91.

Banco de Desarrollo de América Latina (2013). Lineamientos para un código latinoamericano de gobierno corporativo. Recuperado de http://scioteca.caf.com/handle/123456789/555 inclusive de forma fraudulenta. Por último, que los riesgos sean detectados a tiempo, de manera preventiva, por un método de control de los recursos adecuado y ajustado a la realidad de las cooperativas.

Por otro lado, esta investigación permitió validar el instrumento de medición de los constructos, "gobierno corporativo" y "arquitectura de control", con base en los lineamientos del BID, el cual es aplicable al sector cooperativo con 21 variables.

Finalmente, se deja abierta la oportunidad, como futura línea de investigación, la validación del instrumento aplicado a otros sectores empresariales, industriales y de servicios.

Buitrago, H., Betancourt, M. (2013). El gobierno corporativo como pilar fundamental para la sostenibilidad de la economía. Gestión \& Desarrollo, 10(1), 195-205.

Cabero, J., Llorente, M. (2013). La aplicación de juicio de experto como técnica de evaluación de las tecnologías de la información (TIC). Eduweb Revista de tecnología de información y comunicación en educación7(2), 11-22.

Calero, A. (2003). Estadística III. La Habana: Editorial Félix Varela.

Charreaux, G. (1987). La théorie positive de l'agence: une synthese de la litterature. De nouvelles théories pour gérer l'entreprise.

Coriat, B., Weinstein, O. (2011). Nuevas teorías de la empresa. Una revisión crítica. Buenos Aires, Argentina: Lenguaje Claro editora

Crespi, R., \& Gispert, C. (1999). Implications for the governance of Spanish companies. II Foro de Finanzas. Segovia: Block Transfer.

Crespo, G., D Ambrosio, G., Racines, A., Castillo, L. (2016). Cómo medir la percepción de responsabilidad social empresarial en la industria de gaseosas. Yura Relaciones Internacionales, 1-18.

De Arquer, M. (1995). Fiabilidad humana: métodos de cuantificación, juicio de expertos. Centro Nacional de Condiciones de Trabajo. 
García, H., Cortez, K., Rodríguez, M. (2009). Activismo legal como factor de cambio en el gobierno corporativo. Recuperado de http://www.scielo.org.co/pdf/cadm/ v22n38/v22n38a03.pdf

Gómez, G., Zapata, N. (2013). Gobierno corporativo: una comparación de códigos de gobierno en el mundo, un modelo para empresas latinoamericanas familiares y no familiares. Recuperado de http://www.redalyc.org/articulo.oa? id=265429948008

Hair, J., Anderson, R., Tatham, R., \& Black, W. (1995). Multivariate Data Analysis with Readings (5 ed.). London: Prentice Hall Inc.

Hernández, R., Fernández, C., Baptista, P. (2010). Metodología de la investigación. D. F., México: McGraw Hill.

Jensen, M. C., Meckling, W. H. (1976). Theory of the firm: Managerial behavior, agency costs, and ownership structure. Journal of Financial Economics, 3(4), 305-360.

Kendall, M., \& Babington, B. (1939). The Problem of $m$ Rankings. The Annals of Mathematical Stastics, 10(3), 275-287.

Luque, T. (1997). Investigación de marketing. Barcelona, España: Ariel.

Mackenzie, S., Podsakoff, P., \& Ahearne, M. (1998). Some Possible Antecedents and Consequences of In-role and Extra-role
Salespersons Perfomance. Journal of Marketing, 62(3), 87-98.

Mendes, G., Núñez, G., \& Oneto, A. (2009). Gobernanza corporativa y desarrollo de mercados de capitales en América Latina. Recuperado de http://scioteca.caf.com/ handle/123456789/494

Organización para la Cooperación y el Desarrollo Económicos (2004). Principios de gobierno corporativo de la OCDE. Recuperado de https://www.oecd.org/daf/ca/corporategovernanceprinciples/37191543.pdf

Ospina, A. (2013). El gobierno corporativo en Colombia. Recuperado de http://repository.unimilitar.edu.co/bitstream/10654/ 11791/1/El\%20Gobierno\%20Corporativo\%20en\%20Colombia.pdf

PricewaterhouseCoopers (11 de marzo de 2017). COSO ERM Framework. Recuperado de http://www.pwc.com/mx/es/riesgos/ coso-erm-framework.html

Vaca, C., \& Casanova, E. (2014). Auditoría de sistemas basada en riesgos al SNNA de la Senescyt. Quito-Sangolquí, Ecuador: Escuela Politécnica del Ejército.

Van Der Fels-Klerx, I., Goossens, L., Saatkamp, H., \& Horst, S. (2002). Elicitation of quantitative data from a heterogeneous expert panel: formal process and application in animal health. Risk Analisis, 22(1), 67-81. 